\title{
Frequent itemsets mining for database auto-administration
}

\author{
Kamel Aouiche, Jérôme Darmont \\ ERIC/BDD, University of Lyon 2 \\ 5 avenue Pierre Mendès-France \\ 69676 Bron Cedex, FRANCE \\ \{kaouiche, jdarmont\}@eric.univ-lyon2.fr
}

\author{
Le Gruenwald \\ School of Computer Science \\ University of Oklahoma \\ Norman, OK 73019, USA \\ ggruenwald@ou.edu
}

\begin{abstract}
With the wide development of databases in general and data warehouses in particular, it is important to reduce the tasks that a database administrator must perform manually. The aim of auto-administrative systems is to administrate and adapt themselves automatically without loss (or even with a gain) in performance. The idea of using data mining techniques to extract useful knowledge for administration from the data themselves has existed for some years. However, little research has been achieved. This idea nevertheless remains a very promising approach, notably in the field of data warehousing, where queries are very heterogeneous and cannot be interpreted easily. The aim of this study is to search for a way of extracting useful knowledge from stored data themselves to automatically apply performance optimization techniques, and more particularly indexing techniques. We have designed a tool that extracts frequent itemsets from a given workload to compute an index configuration that helps optimizing data access time. The experiments we performed showed that the index configurations generated by our tool allowed performance gains of $15 \%$ to $25 \%$ on a test database and a test data warehouse.
\end{abstract}

\section{Introduction}

Large-scale usage of databases requires a Database Administrator (DBA) whose principal role is data management, both at the logical level (schema definition) and the physical level (files and disk storage), as well performance optimization. With the wide development of Database Management Systems (DBMSs), minimizing the administration function has become critical to achieve acceptable response times even at load peaks [22]. One important DBA task is the selection of suitable physical structures to improve the system performances by minimizing data access time [10].

Indexes are physical structures that allow a direct access to the data. From the DBA's point of view, performance op- timization lies mainly in the selection of indexes and materialized views [4, 12]. These physical structures play a particularly significant role in decision-support databases such as data warehouses due to their huge volume and complex queries.

The problem of selecting an optimal index set for a database has been studied since the seventies. The most recent studies regarding index selection use the DBMS' query optimizer to estimate the cost of various configurations of candidate indexes [3, 7, 8, 11]. However, the idea of using data mining techniques to extract useful knowledge for administration from the data themselves has been around for some years [6]. Little work has been done, though. In this paper, we designed and coded a tool that exploits data mining to recommend a relevant index configuration.

Assuming that index utility is strongly correlated to the usage frequency of the corresponding attributes within a given workload, the search for frequent itemsets [1] appeared well adapted to highlight this correlation and facilitate index selection. Our tool parses the transaction log file (the set of queries executed by the DBMS) to build a context for mining frequent itemsets. This context connects queries from the input workload to the attributes that may be indexed. The output frequent itemsets are sets of attributes forming a configuration of candidate indexes. Finally, various strategies can be applied to select the indexes to effectively build from within this configuration.

In the remainder of this paper, we present our proposal in Section 2 and some preliminary experimental results in Section 3, and then finally conclude the paper and present future research perspectives in Section 4.

\section{Frequent itemsets mining for index selec- tion}

\subsection{Principle}

Our approach exploits the transaction log to extract an index configuration. The queries from the transaction $\log$ 


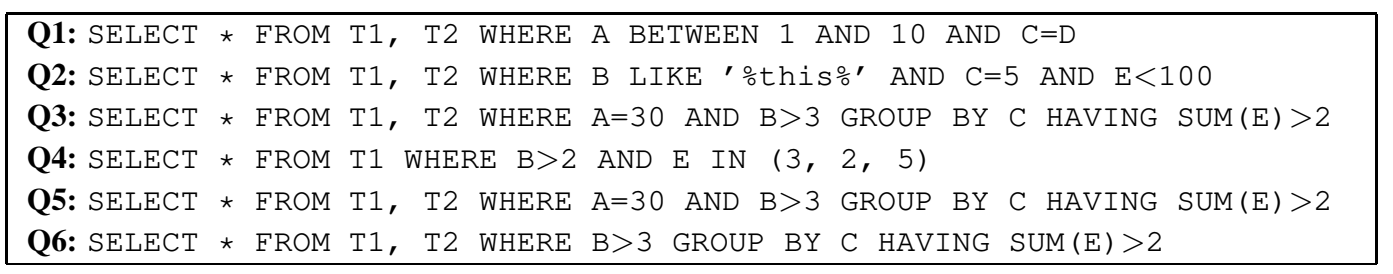

Figure 1. Sample workload

constitute a workload that is treated by an SQL query analyzer. The SQL query analyzer extracts all the attributes that may be indexed (indexable attributes). Then, we build a "query-attribute" matrix, the rows of which are the workload queries, and the columns are the indexable attributes. The role of this matrix is to link each indexable attribute to the workload queries it appears in.

This matrix represents the extraction context for frequent itemsets. To compute these frequent itemsets, we selected the Close algorithm [17, 18], because its output is the set of the frequent closed intemsets (closed regarding the Galois connection [18]), which is a generator for all the frequent itemsets and their support. In most cases, the number of frequent closed itemsets is much lower than the total number of frequent itemsets obtained by classical algorithms such as Apriori [2]. In our context, using Close enables us to obtain a smaller (though still significant) configuration of candidate indexes faster.

Finally, we select from the configuration of candidate indexes (that corresponds to the input workload) the most relevant indexes and create them.

\subsection{Workload extraction}

We assume that a workload similar to the one presented in Figure 1 is available. Such a workload can be easily obtained either from the DBMS' transaction logs, or by running an external application such as Lumigent's Log Explorer [14].

\subsection{Indexable attributes extraction}

To reduce response time when running a database query, it is best to build indexes on the very attributes that are used to process the query. These attributes belong to the WHERE, ORDER BY, GROUP BY, and HAVING clauses of SQL queries [7].

We designed a syntactic analyzer that is able to operate on any SQL query type (selections and updates subqueries are allowed), and extracts all the indexable attributes. This process is applied to all the queries from the workload.

\subsection{Building the extraction context for the fre- quent closed itemsets}

We build a matrix (Figure 2) the rows of which represent the workload queries, and the columns represent the set of all the indexable attributes identified in the previous step. This "query-attribute" matrix links each query to the indexable attributes within it. Attribute presence in a query is symbolized by 1 , and absence by 0 .

\begin{tabular}{|l|c|c|c|c|c|}
\cline { 2 - 7 } \multicolumn{1}{c|}{} & \multicolumn{5}{c|}{ Attributes } \\
\hline Queries & A & B & C & D & E \\
\hline Q1 & 1 & 0 & 1 & 1 & 0 \\
\hline Q2 & 0 & 1 & 1 & 0 & 1 \\
\hline Q3 & 1 & 1 & 1 & 0 & 1 \\
\hline Q4 & 0 & 1 & 0 & 0 & 1 \\
\hline Q5 & 1 & 1 & 1 & 0 & 1 \\
\hline Q6 & 0 & 1 & 1 & 0 & 1 \\
\hline
\end{tabular}

Figure 2. Sample extraction context

\subsection{Frequent closed itemsets mining}

The Close algorithm scans in breadth first a lattice of closed itemsets in order to extract the frequent closed itemsets and their support. Its input is an extraction context such as the one presented in Figure 2

Intuitivelty, a closed itemset is a maximal set of items (attributes) that are common to a set of transactions (queries). For instance, in Figure 2] s extraction context, the BCE itemset is closed because it is the largest set of common attributes for the set of queries $\{\mathrm{Q} 2, \mathrm{Q} 3, \mathrm{Q} 5, \mathrm{Q} 6\}$. On the other hand, the $\mathrm{BC}$ itemset is not closed since all the queries containing attributes B and C (Q2, Q3, Q5, and Q6) also contain attribute E. Eventually, a closed itemset is said frequent when its support is greater or equal to a threshold parameter named minsup (minimal support).

The application of Close on the context presented in Figure 2 outputs the following set of frequent closed itemsets (and their support) for a minimal support equal to $2 / 6$ : $\{(\mathrm{AC}, 3 / 6),(\mathrm{BE}, 5 / 6),(\mathrm{C}, 5 / 6),(\mathrm{ABCE}, 2 / 6),(\mathrm{BCE}, 4 / 6)\}$. 
We consider this set as our configuration of candidate indexes.

\subsection{Indexes construction}

The higher the size of the input workload is, the higher the number of candidate indexes obtained with our approach becomes. Thus, it is not feasible to build all the proposed indexes. Index creation time, and later update time, would both be too costly. Hence, it is necessary to devise filtering methods or processes to reduce the number of indexes to generate.

The first naive method is to build all the candidate indexes. This method is only applicable when the number of indexes is relatively small. In that particular case, creation and update times remain acceptable.

In the context of decision-support databases, and more particularly, of data warehouses, building indexes is a fundamental issue because of the huge volume of data stored in fact tables and some dimension tables. Thus, it is more critical to build indexes on large tables. Index contribution on small tables can indeed prove negligible, and even sometimes, costly.

Statistical input, such as the cardinality of the attributes to be indexed, may also be exploited to build indexes. An attribute's cardinality is the distinct number of values for this attribute within a given relation. Depending on the cardinality, indexing may be more or less efficient. If the cardinality is very large, an index degenerates toward a sequential scan (of the index structure itself); and if it is very small, an index might not bring a very significant improvement [21]. Hence, the best choice might be to build indexes on attributes with an "average" cardinality.

In this first study, we took a particular interest in table sizes. We indeed established two strategies to build indexes from the union of the frequent closed itemsets provided by Close. The first strategy systematically builds all the proposed indexes (naive method). In this case, each frequent closed itemset corresponds to an index to be created. The second strategy takes the size of the tables an index refers to into account. In this case, the DBA must define whether a table is large or not, and only indexes on attributes from these large tables are built.

\subsection{Comparison with the existing methods}

Unlike the index selection methods that have been recently developed, the tool that we propose does not communicate with the DBMS' query optimizer. The communication between the index selection tool and the optimizer is usually costly and must be minimized. An index configuration computing time is indeed proportional to the size of the workload, which is typically large. Our method based on frequent itemsets mining is also greedy in terms of computing time, but it is currently difficult for us to determine which approach generates the heaviest overhead for the system.

However, we are more interested in the quality of the generated indexes. For instance, the Index Selection Tool (IST) developed by Microsoft within the SQL Server DBMS [7] exploits a given workload and provides a configuration of mono-attribute candidate indexes. A greedy algorithm selects the best indexes from this configuration, using estimated costs computed by the query optimizer. The process then reiterates to generate two-attribute indexes using the mono-attribute indexes, and similarly, to generate multiattribute indexes of higher order. By mining frequent closed itemsets, our tool directly extracts a set of mono-attribute and multi-attribute indexes. Hence, we do not build an initial mono-attribute index configuration a priori, and we do not need to use any heuristic to build multi-attribute candidate indexes by successive iterations like IST. We believe that this approach avoids the generation and cost evaluation of irrelevant indexes.

\section{Experiments}

In order to validate our approach, we have applied it on a test database and a test data warehouse. Our objective here is more to find out whether our proposal makes sense in practice than to perform true performance tests.

We have chosen the TPC-R decision-support benchmark [20] for our experiments on a relational database because it is a standard that should allow us to easily compare our approach to the other existing methods in the future. We have generated the TPC-R 1 GB database and used the benchmark's 22 read-only queries (labeled Q1 to Q22). In this first experiment, we suppose refresh operations occur off-line. However, in order to take index management overhead into account, future performance tests will also include TPC-R's RF1 and RF2 refresh functions.

On the other hand, to the best of our knowledge, there is no standard benchmark for data warehouses yet (TPCDS is still in development [19]). Hence, we worked on a small datamart that had been previously developed in our laboratory. This accidentology datamart is composed of an Accident fact table and four dimension tables: Place, Condition, Date and PersonResponsible. It occupies $15 \mathrm{MB}$ on disk. Starting from our previous analyses on this datamart, we also designed a realistic decision-support workload that is specifically adapted to it. This workload includes both selection and update operations. We cannot present it in detail here due to lack of space; interested readers are referred to [5].

Both the TPC-R database and the accidentology datamart have been implanted within the SQL Server 2000 


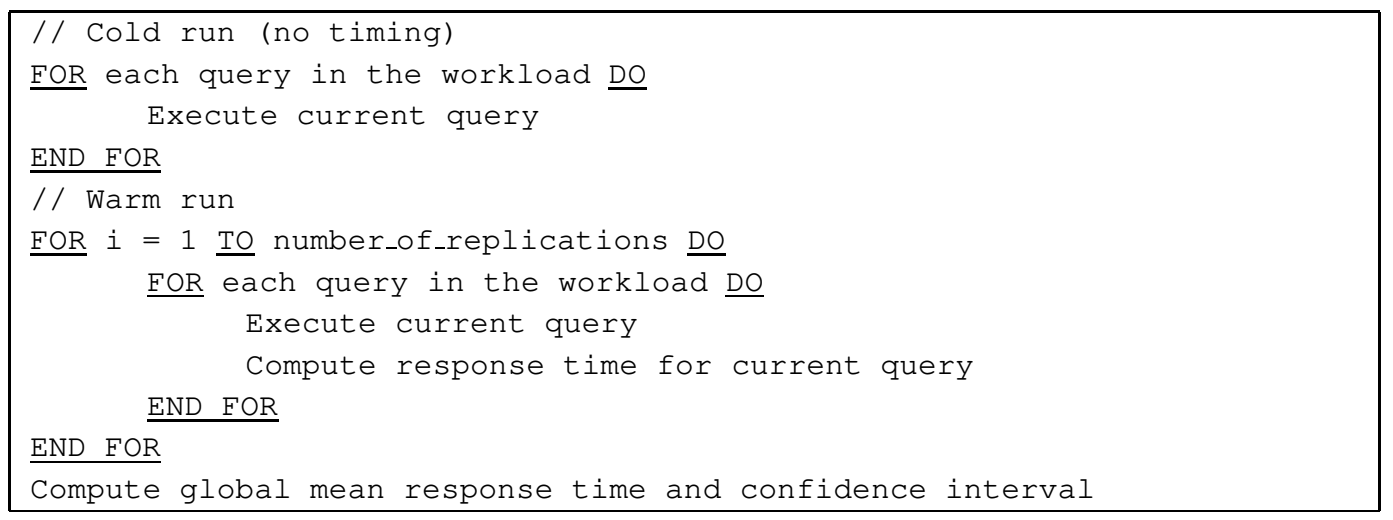

Figure 3. Test protocol

DBMS.

The test protocol we adopted is presented in Figure 3 . This algorithm has been executed for various values of the Close minsup (minimal support) parameter. In practice, this parameter helps us limiting the number of indexes to generate by selecting only those that are the most frequently used by the workload. At each step corresponding to a value of minsup, we compute the mean response time for the input workload.

\subsection{Experiments on TPC-R}

The results we obtained are presented in Figure 4 and 5 . The results from Figure 4 correspond to the creation of all the candidate indexes obtained with Close, while the results of Figure 5 correspond to a filter on this configuration (indexes on large tables only; cf. Section 2.6.

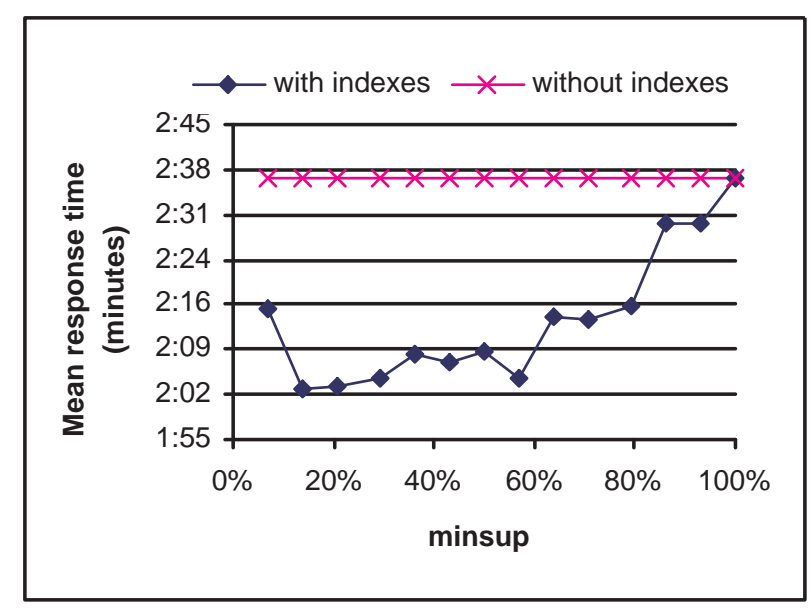

Figure 4. TPC-R results - All indexes

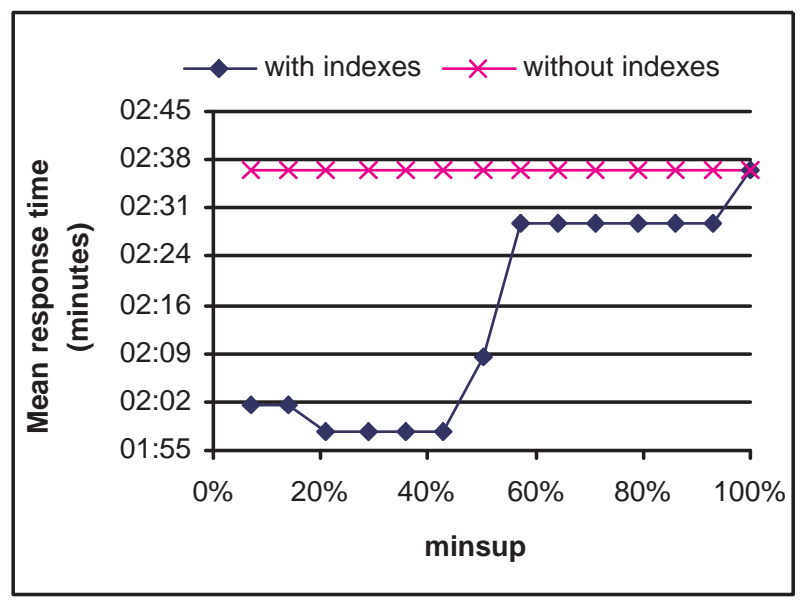

Figure 5. TPC-R results - Indexes on large tables

Figure 4 and 5 show that, in comparison with a sequential scan with no indexes, we achieve a gain in performance for the two strategies regardless of the value of minsup. The maximum response time gain, which is computed as the difference between the mean response time without indexes and the lowest mean response time with indexes, is close to $22 \%$ in the first case and $25 \%$ in the second case. The average response time gains computed over all values of minsup are $14.4 \%$ and $13.7 \%$ in the first and second case, respectively. In the first case, the response time improves until minsup reaches $15 \%$, and then degrades at a steady rate, while in the second case, it remains at its lower value in a broader range (from $20 \%$ minsup to $50 \%$ minsup) before degrading abruptly. The large number of indexes to be generated in the first case can explain this behavior. Considering only indexes associated with large tables helps reducing the number of generated indexes and avoids index creation 
for small tables (since they induce a low benefit).

Finally, for high values of minsup, the mean response time becomes close to that obtained without generating any index in both cases. This was predictable since for a very high minsup, no or very few indexes are actually generated. In the second case, this state is reached sooner since fewer indexes are built, which explains the lower average gain.

\subsection{Data warehouse experiments}

For this series of experiments, we applied the same protocol (Figure 3). However, we did not employ the large table index creation strategy since all the tables in our test datamart have similar sizes.

The results we obtained are presented in Figure 6 The maximum gain in performance is close to $15 \%$ while the average gain is $6.4 \%$. Figure 6 shows that building indexes is actually more costly than not building them for minsup values ranging between $10 \%$ and $25 \%$. This may be explained by the high number of generated indexes, and thus a high index generation time. Furthermore, since the $15 \mathrm{MB}$ datamart is stored completely in the main memory, the indexes are useful only when it is first loaded. In this context, many sparsely used indexes must also be loaded, which penalizes global performance.

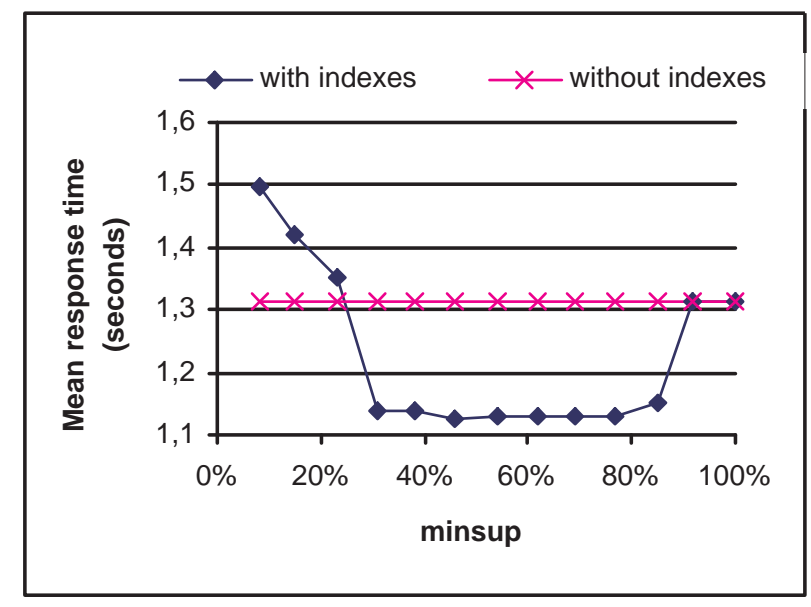

\section{Figure 6. Accidentology datamart results}

The best gain in response time appears for minsup values ranging between $30 \%$ and $85 \%$, when the number of indexes is such that the index generation overhead is lower than the performance increase achieved when loading the datamart. Beyond that point, response time degrades and becomes close to that obtained without indexes because there are a few or no indexes to generate.

Another possible explanation to the lower performances obtained for our datamart, in comparison to the results achieved on the TPC-R database, may come from the structure of the created indexes. Bitmap and star-join indexes are best adapted to data warehouses [15, 16], while the default indexes in SQL Server are variants of B-trees.

\section{Conclusions and perspectives}

We presented in this paper a novel approach for automatic index selection in DBMSs. The originality of our study rests on the extraction of frequent itemsets to determine an index configuration. Indeed, we assume that the importance of an indexable attribute is strongly correlated with its frequency of appearance in the workload's queries. In addition, the use of a frequent itemsets mining algorithm such as Close enables us to generate mono-attribute and multi-attribute indexes on the fly, without having to implement an iterative process that successively creates increasingly large multi-attribute indexes based on an initial set of mono-attribute indexes.

Our first experimental results show that our technique indeed allows response time improvements of $20 \%$ to $25 \%$ for a decision-support workload applied to a relational database (TPC-R benchmark). We also proposed two strategies to carry out an index selection among the candidate indexes: the first strategy systematically creates all the candidate indexes, while the second only creates the indexes that are related to so-called large tables. The second strategy allows better performance improvements because it proposes a better compromise between the space occupied by the indexes (the number of created indexes is limited to those that are defined on attributes from large tables) and the use of creating an index (it is not beneficial to create an index on a small table).

We also performed tests on an accidentology datamart, on which we applied an ad hoc decision-support workload. The gain in response time, about $14 \%$, is less significant than in the case of TPC-R. This can be explained by the fact that the default indexes created by SQL Server are B-tree variants and not bitmap or star-join indexes, which would be better adapted for a data warehouse.

Our study shows that using data mining techniques for DBMS auto-administration is promising. However, it is only a first approach and it opens up many prospects for research. Our first research perspective is to improve index selection by designing more elaborated strategies than the exhaustive use of a configuration or the exploitation of relatively basic information relating to table sizes. A more accurate cost model regarding table features (other than size), or a strategy for weighting the workload's queries (by type of query: selection or update), could help us. The use of other unsupervised data mining methods such as clustering could also provide smaller sets of frequent itemsets.

It also appears essential to test our method further to bet- 
ter evaluate the overhead it generates, both in terms of indexes generation time and maintenance time. In particular, it is necessary to apply it on large data warehouses, while exploiting adapted indexes. It would also be very interesting to compare it in a more systematic way to the IST tool that has been developed by Microsoft, either through complexity computations of the index configuration generation heuristics (overhead), or by experiments aiming at evaluating the quality of these configurations (response time improvement and overhead due to index maintenance).

Extending or coupling our approach with other performance optimization techniques (materialized views, buffer management, physical clustering, etc.) also constitutes a promising research perspective. Indeed, in the context of data warehouses, it is mainly in conjunction with other physical structures (primarily materialized views) that indexing allows the most significant performance gains [3, 4, 12].

Finally, it would also be interesting to study how algorithms for mining functional dependencies [13] or inclusion dependencies [9] in databases might be exploited in our context. Many join operations (natural joins) are indeed carried out following inclusion dependencies (concept of foreign key). Discovering hidden dependencies within the data could thus help us generating relevant indexes or materialized views without needing an input workload.

\section{References}

[1] R. Agrawal, T. Imielinski, and A. N. Swami. Mining association rules between sets of items in large databases. SIGMOD Record, 22(2):207-216, 1993.

[2] R. Agrawal and R. Srikant. Fast algorithms for mining association rules. In 20th International Conference on Very Large Data Bases (VLDB 1994), Santiago, Chile, pages 487-499, 1994.

[3] S. Agrawal, S. Chaudhuri, and V. R. Narasayya. Automated selection of materialized views and indexes in SQL databases. In 26th International Conference on Very Large Data Bases (VLDB 2000), Cairo, Egypt, pages 496-505, 2000.

[4] S. Agrawal, S. Chaudhuri, and V. R. Narasayya. Materialized view and index selection tool for Microsoft SQL Server 2000. 2001 ACM SIGMOD International Conference on Management of Data, Santa Barbara, USA, 2001.

[5] K. Aouiche. Accidentology datamart schema and workload. http://bdd.univ-lyon2.fr/download/chargeaccidentologie.pdf, 2002.

[6] S. Chaudhuri. Data mining and database systems: Where is the intersection? Data Engineering Bulletin, 21(1):4-8, 1998.

[7] S. Chaudhuri and V. R. Narasayya. An efficient cost-driven index selection tool for Microsoft SQL Server. In 23rd International Conference on Very Large Data Bases (VLDB 1997), Athens, Greece, pages 146-155, 1997.
[8] S. Chaudhuri and V. R. Narasayya. Autoadmin 'what-if' index analysis utility. In 1998 ACM SIGMOD International Conference on Management of Data, Seattle, USA, pages 367-378, 1998.

[9] F. De Marchi, S. Lopes, and J.-M. Petit. Efficient algorithms for mining inclusion dependencies. In 8 th International Conference on Extending Database Technology (EDBT 2002), Prague, Czech Republic, volume 2287 of LNCS, pages 464-476, 2002.

[10] S. J. Finkelstein, M. Schkolnick, and P. Tiberio. Physical database design for relational databases. TODS, 13(1):91128, 1988.

[11] M. R. Frank, E. Omiecinski, and S. B. Navathe. Adaptive and automated index selection in RDBMS. In $3 \mathrm{rd}$ International Conference on Extending Database Technology (EDBT 1992), Vienna, Austria, volume 580 of LNCS, pages 277-292, 1992.

[12] H. Gupta. Selection and maintenance of views in a data warehouse. PhD thesis, Stanford University, 1999.

[13] S. Lopes, J.-M. Petit, and L. Lakhal. Efficient discovery of functional dependencies and Armstrong relations. In 7th International Conference on Extending Database Technology (EDBT 2000), Konstanz, Germany, volume 1777 of LNCS, pages 350-364, 2000.

[14] Lumigent Technologies. Log Explorer for SQL Server. http://www.lumigent.com/products/le_sql/le_sql.htm, 2002.

[15] P. O'Neil and G. Graefe. Multi-table joins through bitmapped join indices. SIGMOD Record, 24(3):8-11, 1995.

[16] P. O'Neil and D. Quass. Improved query performance with variant indexes. SIGMOD Record, 26(2):38-49, 1997.

[17] N. Pasquier, Y. Bastide, R. Taouil, and L. Lakhal. Discovering frequent closed itemsets for association rules. In 7 th International Conference on Database Theory (ICDT 1999), Jerusalem, Israel, volume 1540 of LNCS, pages 398-416, 1999.

[18] N. Pasquier, Y. Bastide, R. Taouil, and L. Lakhal. Efficient mining of association rules using closed itemset lattices. Information Systems, 24(1):25-46, 1999.

[19] M. Poess, B. Smith, L. Kollar, and P.-A. Larson. TPC-DS: Taking decision support benchmarking to the next level. In 2002 ACM SIGMOD International Conference on Management of Data, Madison, USA, 2002.

[20] Transaction Processing Council. TPC Benchmark R Standard Specification, 1999.

[21] S. Vanichayobon and L. Gruenwald. Indexing techniques for data warehouses' queries. Technical report, University of Oklahoma, School of Computer Science, 1999.

[22] G. Weikum, A. Monkeberg, C. Hasse, and P. Zabback. Selftuning database technology and information services: from wishful thinking to viable engineering. In 28th International Conference on Very Large Data Bases (VLDB 2002), Hong Kong, China, 2002. 